\title{
Glucocorticoids Play a Fundamental Role in Protecting the Brain during Innate Immune Response
}

\author{
Sylvain Nadeau and Serge Rivest \\ Laboratory of Molecular Endocrinology, Centre Hospitalier de l'Université Laval Research Center and Department of Anatomy and Physiology, Laval \\ University, Québec, Canada G1V 4G2
}

\begin{abstract}
The innate immune system plays a crucial role in protecting the host against infectious microorganisms. An inappropriate control of this system may have profound consequences, because of the maintained production of specific proinflammatory molecules. Glucocorticoids are the most efficient endogenous molecules that provide negative feedback on proinflammatory signaling and gene expression. Here we show that activation of this system is not detrimental for the brain but a profound neurodegeneration takes place in animals treated with the glucocorticoid receptor inhibitor Mifepristone (RU486). This drug increased the inflammatory reaction induced by a single intracerebral bolus of lipopolysaccharide (LPS). Inhibition of tumor necrosis factor $\alpha$ (TNF- $\alpha$ ) totally abolished the neurotoxic effect of the endotoxin, and chronic infusion of the cytokine mimicked the treatment combining RU486 and LPS. The neuronal damage caused by TNF- $\alpha$ is dependent on both nitric oxide and caspase pathways. In controlling the cerebral innate immunity and microglial TNF- $\alpha$ production, glucocorticoids play a major role in protecting the brain against bacterial cell wall components.
\end{abstract}

Key words: caspases; cytokines; gram-negative cell wall component; inflammation; innate immunity; microglia; neurodegeneration; NF- $\kappa$ B; TNF- $\alpha$

\section{Introduction}

Glucocorticoids (GCs) are involved in the regulation of many physiological systems, but inappropriate regulation of this endocrine axis has profound consequences for the organism. One known outcome of elevated GC levels in stressed individuals is exacerbation of the rate of neuronal cell death during normal aging (Sapolsky, 1996). On the other hand, GCs are the most powerful endogenous immunosuppressors, especially for the innate immune response and the subsequent inflammatory reaction (McKay and Cidlowski, 1999). Indeed, GCs are potent inhibitors of transcription of genes encoding most of the proteins involved in the innate immune system, and a large body of evidence suggests that nuclear factor $\kappa \mathrm{B}(\mathrm{NF}-\kappa \mathrm{B})$ is a key step in this process (McKay and Cidlowski, 1999). Activated GC receptors (GCRs) are able to interfere with the transactivation potential of the $\mathrm{p} 65 \mathrm{NF}-\kappa \mathrm{B}$ subunit as well as activator protein 1 (AP-1) (De Bosscher et al., 1997; Wissink et al., 1998). Altogether these effects of GCs ultimately lead to a decrease in proinflammatory signal transduction pathways and gene expression, which is an essential

\footnotetext{
Received Feb. 4, 2003; revised April 9, 2003; accepted April 21, 2003.

This research was supported by the Canadian Institutes of Health Research (CIHR). Sylvain Nadeau holds a Studentship from the CIHR and Serge Rivest is a CIHR Scientist and holds a Canadian Research Chair in Neuroimmunology. We thank Dr. A. Israel (Institut Pasteur, Paris, France) for the mouse $1 \kappa B \alpha C D N A$, Dr. P. Gray (Genentech, South San Francisco, CA) for the plasmid containing the mouse IL-1 $\beta$ CDNA, Dr. K. Pahan (University of Nebraska, Lincoln, NE) for the mouse IL-12p40 CDNA, Dr. Doug Feinstein (University of Illinois, Chicago, IL) for the rat CD14 plasmid, and Dr. T. Itoh (Children's Hospital, Philadelphia, PA) for the rat caspase-8 cDNA. Thanks are extended to Dr. Nicolas Turin, Alain Simard, and Nataly Laflamme for critical analysis of this manuscript.

Correspondence should be addressed to Dr. Serge Rivest, Laboratory of Molecular Endocrinology, Centre Hospitalier de I'Université Laval Research Center, 2705 Boulevard Laurier, Québec, Canada, G1V 4G2. E-mail: Serge.Rivest@crchul.ulaval.ca.

Copyright $\odot 2003$ Society for Neuroscience $\quad$ 0270-6474/03/235536-09\$15.00/0
}

endogenous mechanism to avoid exaggerated responses during immunogenic challenges.

Such fine control of GCs on the innate immune system and the inflammatory reaction may also exist in the CNS. For a long time, the brain was considered to be a privileged organ from an immunological point of view, because of its inability to mount an immune response and process antigens. Although this is partly true, the CNS shows a well organized innate immune reaction in response to systemic bacterial infection and cerebral injuries (for review, see Nguyen et al., 2002). A single systemic injection with the bacterial cell wall component lipopolysaccharide (LPS) causes robust and transient proinflammatory signaling and transcriptional activation of genes encoding cytokines, chemokines, proteins of the complement system, and immune receptors across the cerebral tissue (Nadeau and Rivest, 2000, 2001; Laflamme and Rivest, 2001; Laflamme et al., 2001; Thibeault et al., 2001). This is also the case when the endotoxin is administered directly within the cerebral tissue; this provokes a strong and time-dependent transcriptional activation of inflammatory genes in microglial cells ipsilateral to the site of injection (Nadeau and Rivest, 2002). Such robust innate immune reaction is not associated with neurodegeneration. In contrast, it is likely to be a crucial player for restoring the homeostatic balance in the presence of bacterial cell wall components within the cerebral tissue (Nadeau and Rivest, 2002).

It is possible that endogenous GCs play a crucial role in the fate of the immune system in the brain. Therefore, this study tested the hypothesis that GCs are essential modulators of the innate immune system in the CNS, and alteration of this negative feedback may be associated with cerebral damage. 
Table 1. Plasmids and enzymes used for probe synthesis

\begin{tabular}{|c|c|c|c|c|c|c|}
\hline \multirow[b]{2}{*}{ Probe } & \multirow[b]{2}{*}{ Vector } & \multirow[b]{2}{*}{ cDNA length } & \multicolumn{2}{|c|}{ Enzyme for linearization } & \multicolumn{2}{|c|}{ RNA polymerase } \\
\hline & & & Antisense & Sense & Antisense & Sense \\
\hline TNF- $\alpha$ & pBluescript SK+ & $716 \mathrm{bp}$ & EcoRl & BamHI & T3 & T7 \\
\hline $\mid \kappa \mathrm{B} \alpha$ & pBluescript SK- & $1114 \mathrm{bp}$ & BamHI & HindIII & $\mathrm{T} 7$ & $\mathrm{~T} 3$ \\
\hline IL-1 $\beta$ & $P C R \|$ & $1360 \mathrm{bp}$ & Kpnl & Xhol & $\mathrm{T} 7$ & Sp6 \\
\hline IL-12p40 & pcL-Neo & $1050 \mathrm{bp}$ & Xhol & Notl & $\mathrm{T} 3$ & T7 \\
\hline Caspase-8 & pBluescript SK+ & 1478 bp & Kpnl & BamHI & T3 & T7 \\
\hline
\end{tabular}

\section{Materials and Methods}

Animals. Adult male Sprague Dawley rats (Charles River Canada, St. Constant, Quebec, Canada; 200 gm) were acclimated to standard laboratory conditions ( $14 \mathrm{hr}$ light/10 hr dark cycle; lights on at 6 A.M. and off at 8 P.M.) with ad libitum access to rat chow and water. Animal breeding and experiments were conducted according to Canadian Council on Animal Care guidelines, as administered by the Laval University Animal Care Committee. A total of 168 rats were assigned to four different protocols divided among the treatment and route of administration.

Surgeries and treatments. Animals were anesthetized with an intraperitoneal injection of a mixture ( $1 \mathrm{ml} / \mathrm{kg}$ body weight) of ketamine hydrochloride $(91 \mathrm{mg} / \mathrm{kg})$ and xylazine $(9 \mathrm{mg} / \mathrm{kg})$, and the site of injection was reached stereotaxically (David Kopf Instruments, Tujunga, Ca). With the incisor bar placed at $3.3 \mathrm{~mm}$ below the interaural line (horizontal zero), the coordinates from bregma for the guide cannula (22 gauge; C313G, Plastic One, Roanoke, VA) were $-0.6 \mathrm{~mm}$ anteroposterior, $-3.3 \mathrm{~mm}$ lateral, and $-2.8 \mathrm{~mm}$ dorsoventral. The guide cannula was secured with screws and cranioplastic cement (cranioplastic powder, Plastic One; Dentsply repair material, Dentsply International, York, PA). The rats were then housed individually for a $10 \mathrm{~d}$ recuperation period. During the first $3 \mathrm{~d}$ after the surgery, rats received once daily a subcutaneous injection of $8 \mathrm{ml}$ of Ringer's lactate (Abbott Laboratories, Saint-Laurent, Canada) and $150 \mu$ l ketoprofen (Rhône Mérieux Canada, Victoriaville, Canada).

On the day of the first set of experiments ( $~ 8: 30$ A.M.), rats received one intraperitoneal injection of Mifepristone (RU486) [50 mg/kg/200 $\mu \mathrm{l}$ (Sigma, St. Louis, MO), M-8046, lot 91K1043] diluted in DMSO (Sigma) or vehicle (DMSO). This dose of RU486 was selected on the basis of previous studies showing the efficiency of the drug in preventing the inhibitory feedback of endogenous glucocorticoids on the innate immune response in the brain (Nadeau and Rivest, 2002). Twelve hours after the intraperitoneal injection, an internal cannula (28 gauge, $14 \mathrm{~mm}$ long from the pedestal, C313I; Plastic One) was connected to the guide cannula, and the rats received an intrastriatal infusion of either LPS [5 $\mu \mathrm{g} / 2 \mu \mathrm{l}$ for $2 \mathrm{~min}$, from Escherichia coli, serotype 055:B5 (Sigma), L-2880, lot $31 \mathrm{~K} 4120)$ ] or sterile saline with a microinjection pump (Razel, model A-99, Razel Scientific Instruments, Stanford, CN). The animals $(n=56)$ were conscious and freely moving at all times throughout the procedure and killed $12 \mathrm{hr}, 1.5,3$ and $7 \mathrm{~d}$ after the last injection.

A second protocol was performed to determine the respective contribution of tumor necrosis factor $\alpha$ (TNF- $\alpha$ ) and interleukin $1 \beta$ (IL-1 $\beta$ ) in mediating the effect of LPS in the CNS. Rats were equipped with chronic indwelling cannulas and treated as described except that they received different mixtures of neutralizing antibodies. Solutions containing a rat (r)IL- $1 \beta$ neutralizing antibody $(2 \mu \mathrm{g} / 5 \mu \mathrm{l}$ for $2 \mathrm{~min}$; R \& D Systems, Minneapolis, MN; AF-501-NA, lot YRO3), an rTNF- $\alpha$ neutralizing antibody ( $2 \mu \mathrm{g} / 5 \mu \mathrm{l}$ for $2 \mathrm{~min}$; R \& D Systems; AF-510-NA, lot XIO3), a mixture of both neutralizing antibodies ( $4 \mu \mathrm{g} / 5 \mu \mathrm{l}$ for $2 \mathrm{~min}$ ), or vehicle were infused in the dorsal basal ganglia $10 \mathrm{hr}$ before the endotoxin challenge via the same route. These neutralizing antibodies were infused a second time $10 \mathrm{hr}$ after the intrastriatal LPS treatment, and rats $(n=56)$ were killed 3 and $7 \mathrm{~d}$ later.

The third series of experiments verified the effects of a chronic infusion of recombinant rat (rr)TNF- $\alpha$ in the rat cerebral tissue. The chronic indwelling cannula was implanted as described before. After the recovery period, a mini-osmotic pump (Alzet, model 2004, Durect Corporation,
Cupertino, CA) was connected to the guide cannula with Intramedic polyethylene tubing (PE-50, inner diameter $0.58 \mathrm{~mm}$, outer diameter $0.965 \mathrm{~mm}$; Dow Corning, Midland, MI) and implanted subcutaneously in the interscapular region. The pumps were filled with either vehicle solution [sterile saline $+0.1 \%$ BSA, ICN Bovine Albumin Fraction V) + $0.01 \%$ L-ascorbic acid (Sigma) or rrTNF- $\alpha(0.0052 \mu \mathrm{g} / 0.26 \mu \mathrm{l}$ per hour; $\mathrm{R} \& \mathrm{D}$ Systems, $510-\mathrm{RT}$, lot AGM010071) and incubated at $37^{\circ} \mathrm{C}$ in sterile saline solution $24 \mathrm{hr}$ before the implantation in vivo. The animals were killed 3, 7, 14, and $21 \mathrm{~d}$ after implantation of the mini-osmotic pumps. Three or five rats were used for the intraparenchymal infusion of vehicle or rrTNF- $\alpha$, respectively, for a total of 32 animals in these experiments.

A fourth set of experiments was performed to investigate the mechanisms mediating the cytotoxic effects of TNF- $\alpha$. Here, the mini-osmotic pumps were filled with vehicle solution [0.1 м PIPES, pH 7.0 (Sigma) + $10 \%$ DMSO, the nonselective inhibitor of the nitric oxide synthase (NOS) N(G)-nitro-L-arginine methyl ester (L-NAME) $(4.167 \mu \mathrm{g} / 0.26 \mu \mathrm{l}$ per hour; Sigma) ], a mixture of caspase-2 (Calbiochem) and caspase- 8 (Calbiochem) inhibitors $(3.125 \mu \mathrm{g} / 0.26 \mu \mathrm{l}$ per hour), or a mixture of the three drugs with and without TNF- $\alpha(0.0052 \mu \mathrm{g} / 0.26 \mu \mathrm{l}$ per hour $)$. The animals $(n=24)$ were killed $14 \mathrm{~d}$ after the beginning of the infusion into the dorsal basal ganglia.

Brain preparation and in situ hybridization histochemistry. Animals were deeply anesthetized after the different treatments with an intraperitoneal injection $(500 \mu \mathrm{l})$ of a mixture of ketamine hydrochloride and xylazine and then rapidly perfused transcardially with $0.9 \%$ saline, followed by $4 \%$ paraformaldehyde in $0.1 \mathrm{M}$ sodium phosphate buffer, $\mathrm{pH}$ 7.4 , at $4^{\circ} \mathrm{C}$. Brains were removed from the skull, postfixed for $2 \mathrm{hr}$, and then placed in $20 \%$ sucrose diluted in $4 \%$ paraformaldehyde-sodium phosphate buffer for $12-15 \mathrm{hr}$. The brains were mounted on a microtome (Reichert-Jung, Cambridge Instruments Company, Deerfield, IL), frozen with dry ice, and cut into $30 \mu \mathrm{m}$ coronal sections from the olfactory bulb to the end of the medulla. The slices were collected in a cold cryoprotectant solution $(0.05 \mathrm{~m}$ sodium phosphate buffer, $\mathrm{pH} 7.3,30 \%$ ethylene glycol, $20 \%$ glycerol) and stored at $-20^{\circ} \mathrm{C}$.

The riboprobes used in this study are described in Table 1; in situ hybridization using ${ }^{35} \mathrm{~S}$-labeled cRNA probes was accomplished as described previously (Laflamme et al., 1999; Nadeau and Rivest, 2000).

Detection of demyelination, cell death, and apoptosis. Demyelination was determined via Luxol Fast Blue (LFB) staining. Every sixth section of the whole rostrocaudal extent of each brain was mounted onto poly-Llysine-coated slides, dried overnight under vacuum, dehydrated through graded concentrations of alcohol (50, 70, and 95\%, 1 min each), and incubated at $60^{\circ} \mathrm{C}$ for $6 \mathrm{hr}$ in LFB solution [Solvent Blue $381 \%$ (Sigma) in $95 \%$ ethanol and $0.5 \%$ acetic acid]. The sections were then rinsed in $95 \%$ alcohol ( $1 \mathrm{~min}$ ), $0.05 \%$ lithium carbonate (Sigma; $1-5 \mathrm{~min}$ ), and $70 \%$ alcohol (two dips). Thereafter, the slides were incubated in $1 \%$ eosine Y solution (EM Diagnostic Systems, Gibbstown, NJ) for $40 \mathrm{sec}$, rinsed in distillated water, incubated in $0.25 \%$ cresyl violet (Sigma) for 40 $\mathrm{sec}$, rinsed in distillated water, dehydrated through graded concentrations of alcohol (50,70, 95, and 100\%; 1 min each), cleared in xylene for $1 \mathrm{~min}$ (two times), and coverslipped with distrene plasticizer xylene (DPX) (Electron Microscopy Sciences, Fort Washington, PA).

Apoptotic cells were labeled by immunohistochemistry using a cleaved caspase-3 monoclonal antibody. Brain sections were washed in sterile DEPC-treated $50 \mathrm{~mm}$ potassium PBS (KPBS) and incubated $48 \mathrm{hr}$ at $4^{\circ} \mathrm{C}$ with the cleaved caspase-3 antibody Asp 175 (Cell Signaling Technology, 
Mississauga, Ontario, Canada), which was diluted in sterile KPBS (1:500) $+0.4 \%$ Triton X-100 $+1 \%$ BSA (fraction V, Sigma). After incubation with the primary antibody, brain slices were rinsed in sterile KPBS and incubated with a mixture of KPBS $+0.02 \%$ Triton X-100 + 1\% BSA + Cy2-conjugated anti-rabbit IgG antibody (1:1500; Jackson ImmunoResearch Laboratory, West Grove, PA) for $3 \mathrm{hr}$ in a dark room at $20^{\circ} \mathrm{C}$. Tissues were thereafter rinsed in sterile KPBS, mounted onto poly-Llysine slides, and coverslipped with a polyvinyl alcohol (Sigma) mounting medium containing 2.5\% 1,4-diazabicyclo(2,2,2)-octane (Sigma) in buffered glycerol (Sigma).

Cell death by apoptosis was also detected via a TdT-FragEL DNA Fragmentation Detection Kit (Oncogene Research Products, San Diego, CA). Positive controls were generated from brain sections of animals that received only sham treatments. These sections were mounted on the slides and covered with $1 \mu \mathrm{g} / \mu \mathrm{l}$ DNase I in $1 \times \mathrm{TBS} / 1 \mathrm{~mm} \mathrm{MgSO}_{4}$ for 20 min at room temperature.

Neuronal death was labeled with the Fluoro-Jade B (FJB) method. Briefly, every sixth section of the whole rostrocaudal extent of each brain was mounted onto poly-L-lysine-coated slides, dried under vacuum for 2 $\mathrm{hr}$, dehydrated through graded concentrations of alcohol (50, 70, and $100 \%, 1 \mathrm{~min}$ ), and rehydrated through graded concentrations of alcohol $(100,70$, and $50 \%, 1 \mathrm{~min}$ each) and $1 \mathrm{~min}$ in distillated water. They were then dipped into and shaken in potassium permanganate $(0.06 \%)$ for 10 $\mathrm{min}$, rinsed $1 \mathrm{~min}$ in distillated water, and dipped into and shaken in a solution containing Fluoro-Jade B [Fluoro-Jade B 0.0004\% (Histochem, Jefferson, AR) + acetic acid $0.1 \%$ (Sigma) + DAPI 0.0002\% (Molecular Probes Eugene, OR)] for $20 \mathrm{~min}$. The slides were thereafter rinsed three times in distillated water (1 min each), dried, dipped in xylene three times (2 min each), and coverslipped with DPX.

Nissl stain was also used as a general index of cellular morphology that may be altered in response to the different treatments.

Staining of infiltrating cells. The Wright stain was used to visualize infiltrating cells within brain parenchyma, and their identification was based on the morphology and color of cytoplasm, nucleus, and granulations. Every sixth section of the whole rostrocaudal extent of each brain was mounted onto poly-L-lysine-coated slides, dried under vacuum for 1 hr, and covered with $2 \mathrm{ml}$ of Wright staining solution (Bayer Corporation Diagnostics Division, Elkhart, IN) for $90 \mathrm{sec}$. They were then covered with an additional $2 \mathrm{ml}$ of buffer solution for $3 \mathrm{~min}$ and washed with the rinse solution provided by the company. Sections were immediately dipped in Xylene and coverslipped with DPX-mounting medium.

Quantitative analysis. Hybridization signals were quantified on x-ray films (Biomax). Briefly, transmittance values (optical density) of the hybridization signal were measured under a Northern Light Desktop Illuminator (Imaging Research) using a Sony Camera Video System attached to a Micro-Nikkor $55 \mathrm{~mm}$-Vivitar extension tube set coupled to a computer and NIH Image software version 1.59/ppc [written by W. Rasband (National Institutes of Health) and available from the internet by anonymous ftp from http://rsb.info.nih.gov/nih-image/download.html]. Optical density (O.D.) values for each pixel were calculated using a known standard of intensity and distance measurements from a logarithmic specter adapted from Bioimage Visage 110s (Millipore, Ann Arbor, MI). The entire hemisphere ipsilateral to the injection site was digitized and subjected to densitometric analysis, yielding measurements of mean density per area. The O.D. of each hemisphere was then corrected for the average background signal by subtracting the O.D. of area without positive signal located in the contralateral side. All measurements were performed in triplicate. Data were reported as mean O.D. values ( \pm SEM).

Demyelination was evaluated on LFB-stained sections that were digitized with an RT-SPOT camera (Diagnostic Instruments, Sterling Heights, MI) mounted directly onto a microscope (BX-60, Olympus) and connected to a computer (PowerMac G4, Macintosh, Apple Computer). The O.D. of corpus callosum and caudoputamen was measured using the NIH Image software, and values of the contralateral side were subtracted from those of the ipsilateral side of three different rostrocaudal levels.

The necrotic area was measured with the NIH Image software, and the data are reported as arbitrary units, whereas the number of FJB- and cleaved caspase-3-positive cells was calculated manually. An area of
$250 \times 250 \mathrm{~mm}^{2}$ was drawn on the computer monitor, and the number of positive cells was counted manually and reported as the mean number of positive cells per $\mathrm{mm}^{2}( \pm \mathrm{SEM})$.

The statistical analyses for the different dependent variables were performed by a two-way ANOVA followed by a Bonferroni/Dunn test procedure as post hoc comparison. Please see the figure legends for more specific details.

\section{Results}

\section{Innate immune response in the site of injection}

A single intrastriatal infusion of LPS caused profound transcriptional activation of numerous genes involved in the control of the innate immune response. Representative examples of such phenomena are depicted in Figure 1. There was no positive signal in the CNS of rats that received an intraperitoneal injection with either the vehicle solution (DMSO) or RU486 before the intrastriatal infusion of saline. However, TNF- $\alpha$-, CD14-, IL- $1 \beta-$, and $\mathrm{I} \kappa \mathrm{B} \alpha$-expressing cells were found at the edge of the tract of the cannula, especially at time $12 \mathrm{hr}$ after saline injection. In contrast to this highly localized pattern, the hybridization signal for most of the transcripts assessed in this study was intense and widespread $12 \mathrm{hr}$ after the single LPS infusion into the dorsal basal ganglia (Fig. 1). Although the hybridization signals for the genes encoding CD14 and caspase- 8 were maximal only at time $36 \mathrm{hr}$ and $3 \mathrm{~d}$, respectively, all transcripts returned to background levels $7 \mathrm{~d}$ after the intracerebral treatment with the bacterial endotoxin (Fig. 1, bottom panels).

Inhibition of GCRs changed the inflammatory reaction provoked by the single LPS bolus. At time $12 \mathrm{hr}$ after intrastriatal LPS infusion, the proinflammatory genes were strongly induced in the brain of rats pretreated or not with RU486, and the signal intensity was similar between both groups of animals. However, expression levels of the immune transcripts were significantly higher in the brain of rats that received RU486 before the intraparenchymal bolus of LPS and killed $3 \mathrm{~d}$ later. The hybridization signals for CD 14 and $\mathrm{I} \kappa \mathrm{B} \alpha \mathrm{mRNAs}$ were still positive $7 \mathrm{~d}$ after the single LPS injection into the dorsal basal ganglia of animals pretreated with the GCR inhibitor (Fig. 1).

\section{Effect of RU486 on cerebral integrity}

The data that RU486 is able to exacerbate the innate immune response in the brain were expected, because GCs act as the critical endogenous negative feedback for the proinflammatory signal transduction pathways and gene transcription. Without such feedback, a single LPS bolus became highly toxic for the cerebral tissue (Fig. 2). Despite the strong and transient inflammatory reaction in regions ipsilateral to the site of infusion, the endotoxin did not affect the cerebral tissue that was evaluated via numerous approaches. In contrast, LPS provoked a nonspecific cell death in animals pretreated with RU486. A localized wound was observed at the site of infusion $36 \mathrm{hr}$ after the treatment combining RU486 with LPS, but the extent of the tissue damage was variable among animals. The fluorochrome FJB, a sensitive and reliable marker for the histochemical localization of neuronal degeneration, was already apparent $36 \mathrm{hr}$ after the insult. The magnitude and intensity of FJB signal increased dramatically $3 \mathrm{~d}$ after LPS administration in rats pretreated with the drug inhibiting GCRs. This was also the case for apoptotic cells that were labeled via a TdT-FragEL DNA fragmentation detection kit and by immunohistochemistry using an antibody directed against anti-cleaved caspase-3 (Table 2, Fig. 2C,D). Cleaved caspase-3immunoreactive signal was already robust in neurons ipsilateral to the site of LPS infusion at time $36 \mathrm{hr}$ after LPS and RU486 
12 hours
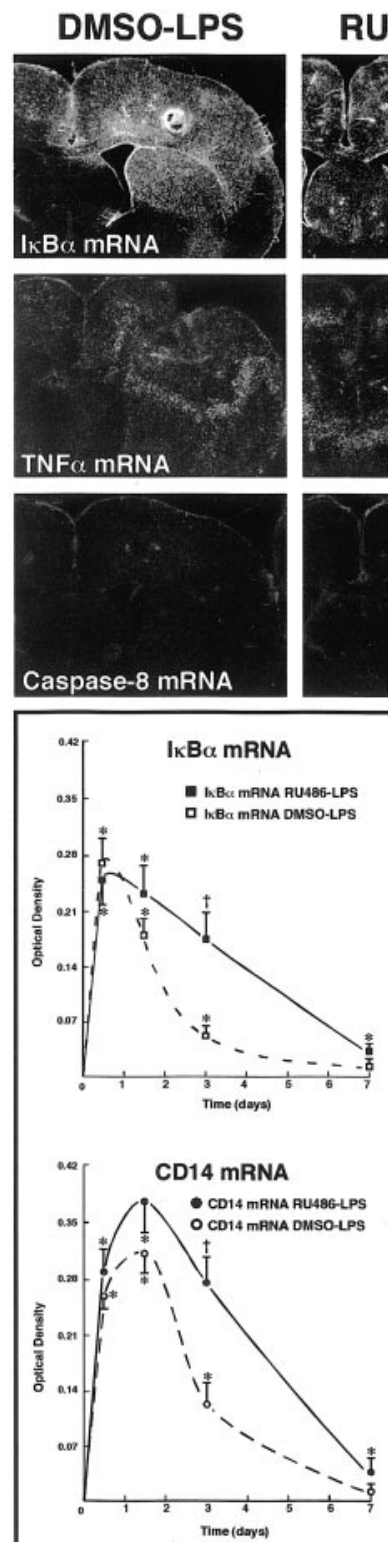

RU486-LPS
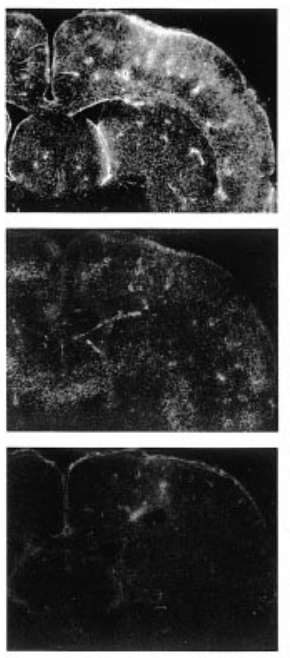
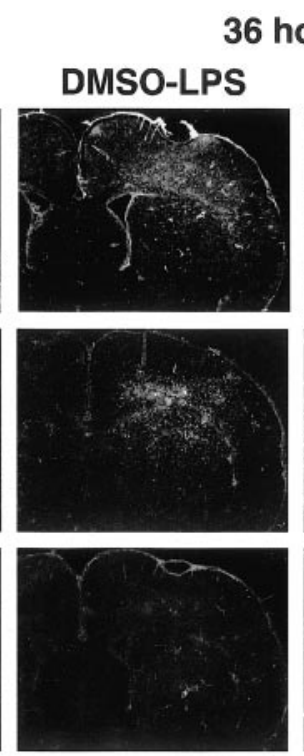

\section{rs}

\section{RU486-LPS}
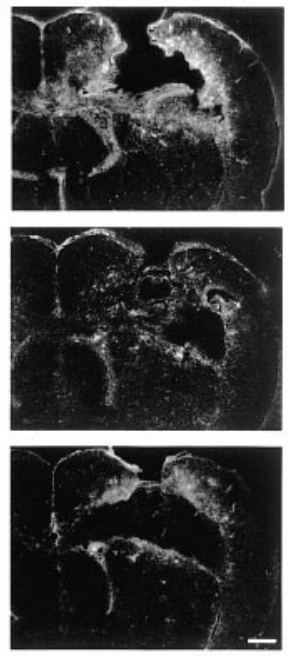
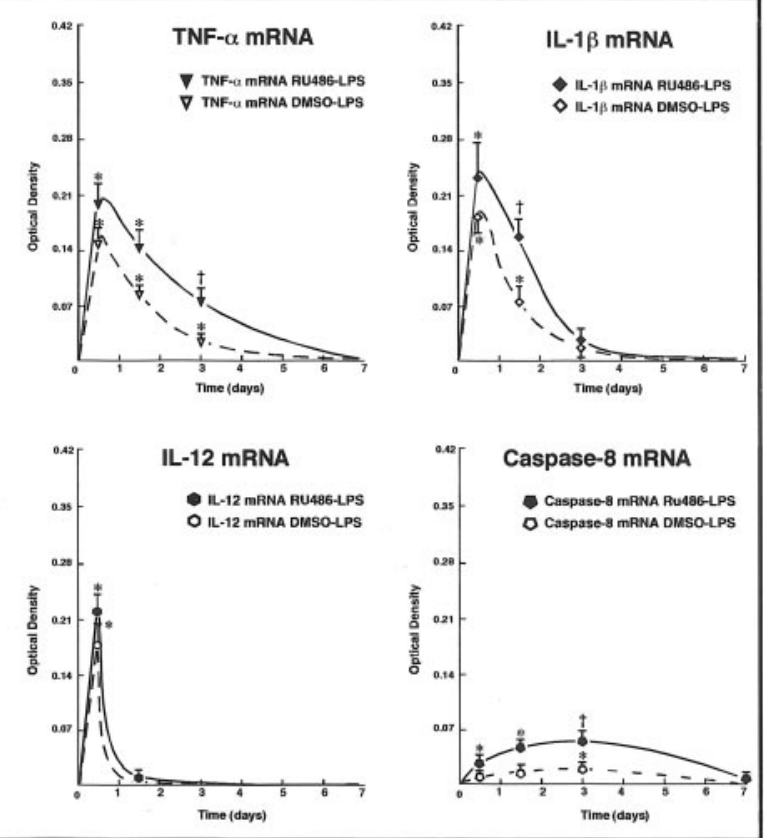

Figure 1. Time-related induction of genes encoding proinflammatory proteins and caspase-8 in the brain of rats that received a single bolus of lipopolysaccharide (LPS) in the dorsal striatum. Animals received a systemic injection with vehicle (DMSO) or the glucocorticoid receptor inhibitor RU486 $12 \mathrm{hr}$ before the intraparenchymal challenge with the endotoxin. The dark-field photomicrographs were taken from brain coronal sections hybridized with different radioactive riboprobes and dipped into nuclear emulsion milk (Kodak). The pictures of the first row depict representative examples of the expression pattern of the inhibitory factor $\kappa \mathrm{B} \alpha(\mathrm{I} \kappa \mathrm{B} \alpha$; index of NF- $\kappa \mathrm{B}$ activity) transcript 12 and $36 \mathrm{hr}$ after an intracerebral LPS bolus in rats that were pretreated systemically with the vehicle DMSO or RU486. Those of the second row show examples of tumor necrosis factor $\alpha$ (TNF- $\alpha$ )-expressing cells, whereas coronal sections of the third row were hybridized with a rat caspase-8cRNA probe. The 0.D. was measured on $x$-ray films (Biomax, Kodak) from digitalized sections and subjected to densitometric analysis using a logarithmic specter of standardized 0.D. yielding measurements of mean density per area. For more information on image analysis, see Materials and Methods. Results represent mean \pm SEM for two (intrastriatal vehicle) or five (intrastriatal LPS) animals per group. Data were analyzed by a two-way ANOVA, followed by a Bonferroni/Dunn test procedure as post hoc comparison (Statview 4.01). There was a significant interaction between the two main independent variables. ${ }^{*} p<0.05$ from their corresponding DMSO/ vehiclegroups; ${ }^{\dagger} p<0.05$ from their corresponding RU486/vehicle and DMSO/LPS groups. Magnification, 3.125×. Scale bar, $1000 \mu \mathrm{m}$. rostrocaudal level. It is nevertheless possible that the backflow of the injected solution explains the more localized damage in the cortex.

\section{Role of cytokines in mediating \\ LPS-induced neurotoxicity}

These results provide solid anatomical evidence that the release of GCs is a critical mechanism in protecting the cerebral tissue when infused with a cell wall component derived from gram-negative bacteria. The genes encoding TNF- $\alpha$ and IL- $1 \beta$ are strongly induced in the brain of rats challenged with RU486 and LPS, and both cytokines have the ability to stimulate apoptotic pathways in various types of cells. We therefore tested whether these cytokines may be involved in the effects of LPS in the brain of rats pretreated with RU486. To do so, rats were infused with an rIL- $1 \beta$ neutralizing antibody, an rTNF- $\alpha$ neutralizing antibody, or a combination of both antibodies in the brain. The injured surface was significantly smaller in the brain of rats that received the antiIL- $1 \beta$ before the endotoxin, but inhibition of this cytokine failed to completely prevent the neurotoxicity caused by the combined RU486 and LPS treatment (Fig. 3). The corpus callosum was still strongly affected by the endotoxin in rats injected with the anti-rIL- $1 \beta$ neutralizing antibody (Fig. $3 A$ ). In contrast, inhibition of TNF- $\alpha$ essentially abolished the neurotoxic effects of LPS. The cerebral tissues of rats that received the neutralizing antibody against TNF- $\alpha$ before the single intrastriatal bolus of LPS was comparable with those of saline-injected animals. The fluorescent signal for both FJB and anti-cleaved caspase- 3 was detectable only in a few cells in the LPS-infused area of rats pretreated with anti-TNF- $\alpha$ (Fig. $3 C, D$ ). The treatment combining both cytokine antibodies before the intraparenchymal LPS infusion in RU486-administered rats also mostly prevented the endotoxin from provoking neuronal damage, but the tissues of these animals were mostly comparable with those of rats pretreated only with the anti-TNF before the cerebral insult.

\section{Mechanisms involved in the effects of TNF- $\alpha$}

To this point, our data indicate that IL- $1 \beta$ and TNF- $\alpha$ are involved in the neurotoxic effects of LPS, but the latter cytokine is more critical in inducing cell death. We consequently investigated whether a chronic infusion of TNF- $\alpha$ may have the ability to mimic the combined treatment of RU486 with intrastriatal treatment (Fig. 2D). The progressive change in neuronal integrity was accompanied by an influx of infiltrating cells, mainly neutrophils, lymphocytes, and monocytes (Fig. 2E).

It is interesting to note that the striatum seems more resistant than the cortex to LPS in RU486-treated rats, but this phenomenon was quite variable among animals, the injection site, and the 
LPS injection. Rats were equipped with an indwelling cannula connected to a miniosmotic pump to deliver the cytokine for a period of 14 consecutive days. Figure 4 depicts representative examples of the target area of rats killed 3, 7, and $14 \mathrm{~d}$ after the chronic infusion of either vehicle solution or $\operatorname{rrTNF}-\alpha$. The chronic intrastriatal TNF- $\alpha$ infusion caused a progressive destruction of cerebral tissue, whereas the brain of saline-infused rats remained intact except for the lesion performed by thecannula. Neuronal apoptosis was also detected by means of immunohistochemistry using an antibody directed against the anti-cleaved caspase- 3 and DNA fragmentation (Table 3, Fig. 4C,D). Similarly to the LPS and RU486 treatment, a mixed population of cells infiltrated the damaged area of TNF- $\alpha$ administered rats. Indeed, numerous monocytes, neutrophils, and lymphocytes were found in the degenerating regions, especially between days 7 and 14 of chronic infusion.

To investigate the mechanisms involved in the cytotoxic effects of TNF- $\alpha$, rats were equipped with a mini-osmotic pump filled with TNF- $\alpha$ mixed with the nonselective inhibitor of NOS L-NAME and inhibitors of caspase-2 and -8. The chronic infusion of TNF- $\alpha$ into the dorsal basal ganglia provoked tissue necrosis, demyelination, neuronal death, and cellular apoptosis (Fig. 5). L-NAME had the ability to significantly reduce the number of FJB-positive neurons, but the NOS inhibitor did not prevent the cytokine from inducing tissue damage and apoptosis. On the other hand, the drug inhibiting caspase- 2 and -8 significantly decreased the necrotic area and the number of both groups of FJB and cleaved caspase-3 cells in response to the chronic TNF infusion. The mixture of both NOS and caspase inhibitors had similar effects and failed to totally abolish TNF- $\alpha$-induced neurotoxicity.

\section{Discussion}

The present set of data provides solid evidence that GCs play a critical role in the control of the innate immune system in the brain. Without such control, activation of this system has profound consequences for the cerebral elements and provokes irreversible damage. A single intraparenchymal bolus of LPS was able
DMSO-LPS (3 days)

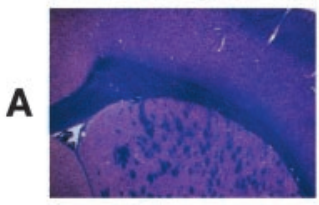

B

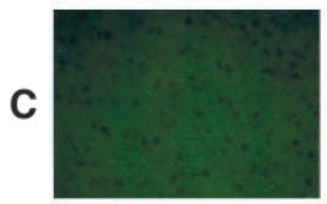

D

E

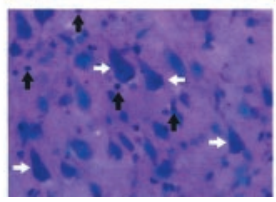

RU486-LPS (12 hours)
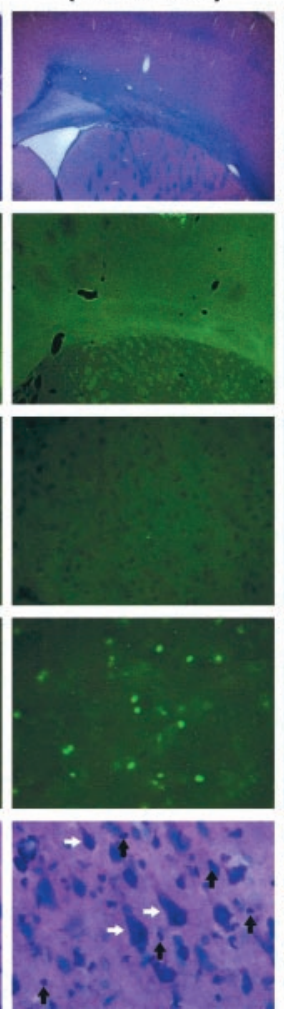

RU486-LPS (1.5 days)
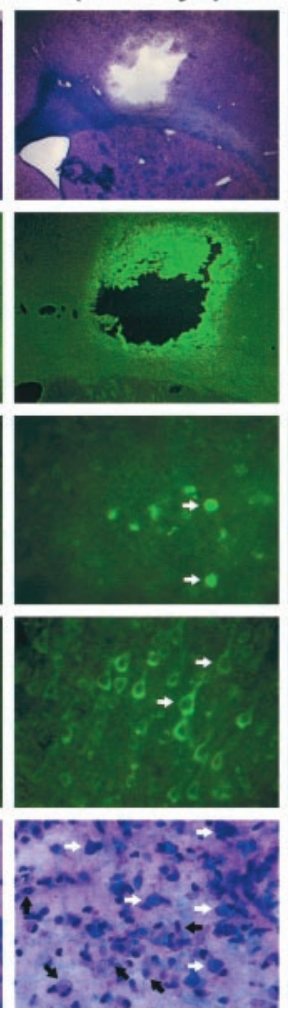

RU486-LPS (3 days)
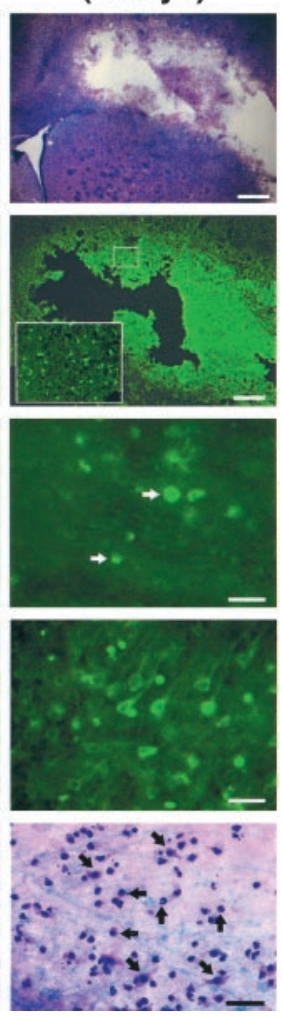

Figure 2. A single intraparenchymal lipopolysaccharide (LPS) injection causes a major neurodegeneration in rats pretreated with RU486. Animals received an intraperitoneal injection of either vehicle solution (DMSO) or RU486 (50 mg/kg) $12 \mathrm{hr}$ before the intracerebral infusion of either vehicle solution (saline) or LPS (5 $\mu \mathrm{g} / 2 \mu \mathrm{l}$ for $2 \mathrm{~min}$ ). $A$, Panels depict representative examples of demyelination and necrotic tissues stained with Luxol Fast Blue. $B$, Panels show examples of Fluoro-Jade B staining used here as index of neurodegeneration. Apoptotic cells are depicted by the images in the panels in C (DNA fragmentation) and D (immunoreactive cleaved caspase-3 cells). The nonspecific staining is caused by the primary antisera, because no signal was found in tissues (from both control and treated rats) incubated without primary antibody. This staining is unlikely to reveal cleaved caspase- 3 in control brains, because this enzyme and its substrates are not located in the nucleus but in the cytoplasm, as depicted by the apoptotic cells of the two right panels in D. E, Panels show the progressive destruction of the extracellular matrix, modification of the neuronal morphology, and appearance of infiltrating cells detected by means of the LFB staining. All photomicrographs were taken at the same rostrocaudal level. Black arrows indicate infiltrating cells; white arrows indicate neurons. Magnification: $A, B$, $3.125 \times ; C-E, 100 \times$. Scale bars: $A, B, 1000 \mu \mathrm{m} ; C-E, 100 \mu \mathrm{m}$.

to increase the transcription of various genes involved in the innate immune response, and such a phenomenon did not alter the neuronal integrity, which was verified via a number of approaches. Cell death induced by apoptosis was never observed in the brain of LPS-treated animals. Other histological straining procedures also failed to provide anatomical evidence that LPS and its induced inflammatory reaction have detrimental effects. Cell bodies, dendrites, and fibers were not changed in regions depicting the robust expression wave of immune genes, including the basal

Table 2. Quantification of brain damage caused by the intraparenchymal LPS infusion in rats pretreated with the glucocorticoid receptor inhibitor RU486

\begin{tabular}{lllcccc}
\hline & Condition & & & \\
\cline { 2 - 6 } & DMSO-Veh (3 d) & DMSO-LPS (3 d) & RU486-Veh (3 d) & RU486-LPS (12 h) & RU486-LPS (1.5 d) & RU486-LPS (3 d) \\
\hline Demyelination (differential 0.D.) & $0.010 \pm 0.001$ & $0.011 \pm 0.003$ & $0.017 \pm 0.005$ & $0.026 \pm 0.029$ & $0.094^{*} \pm 0.041$ & $0.198^{* \dagger} \pm 0.024$ \\
Necrotic area (arbitrary units) & $2.81^{a} \pm 0.67$ & $2.66^{a} \pm 0.43$ & $2.87^{a} \pm 0.72$ & $4.14 \pm 0.45$ & $18.43^{*} \pm 3.46$ & $41.52^{*} \pm 8.89$ \\
TdT-FragEL (number of cells/mm $/ \mathrm{mm}^{2}$ ) & $0 \pm 0$ & $0 \pm 0$ & $0 \pm 0$ & $0 \pm 0$ & $186^{*} \pm 24$ & $224^{*} \pm 39$ \\
Cleaved caspase-3 (number of cells/mm²) & $0 \pm 0$ & $0 \pm 0$ & $0 \pm 0$ & $0 \pm 0$ & $703^{*} \pm 82$ \\
\hline
\end{tabular}

DMSO-Veh, Intraperitoneal injection of DMSO and intrastriatal (IS) infusion of saline; DMSO-LPS, intraperitoneal DMSO injection and IS LPS infusion; RU486-Veh, intraperitoneal RU486 injection and IS saline infusion; RU486-LPS, intraperitoneal RU486 injection and IS LPS infusion. ${ }^{*} p<0.05$ from the RU486/Veh-treated group; ${ }^{\dagger} p<0.05$ from the RU486/LPS-treated group at time $1.5 \mathrm{~d}$.

${ }^{a}$ Tract of cannula. 

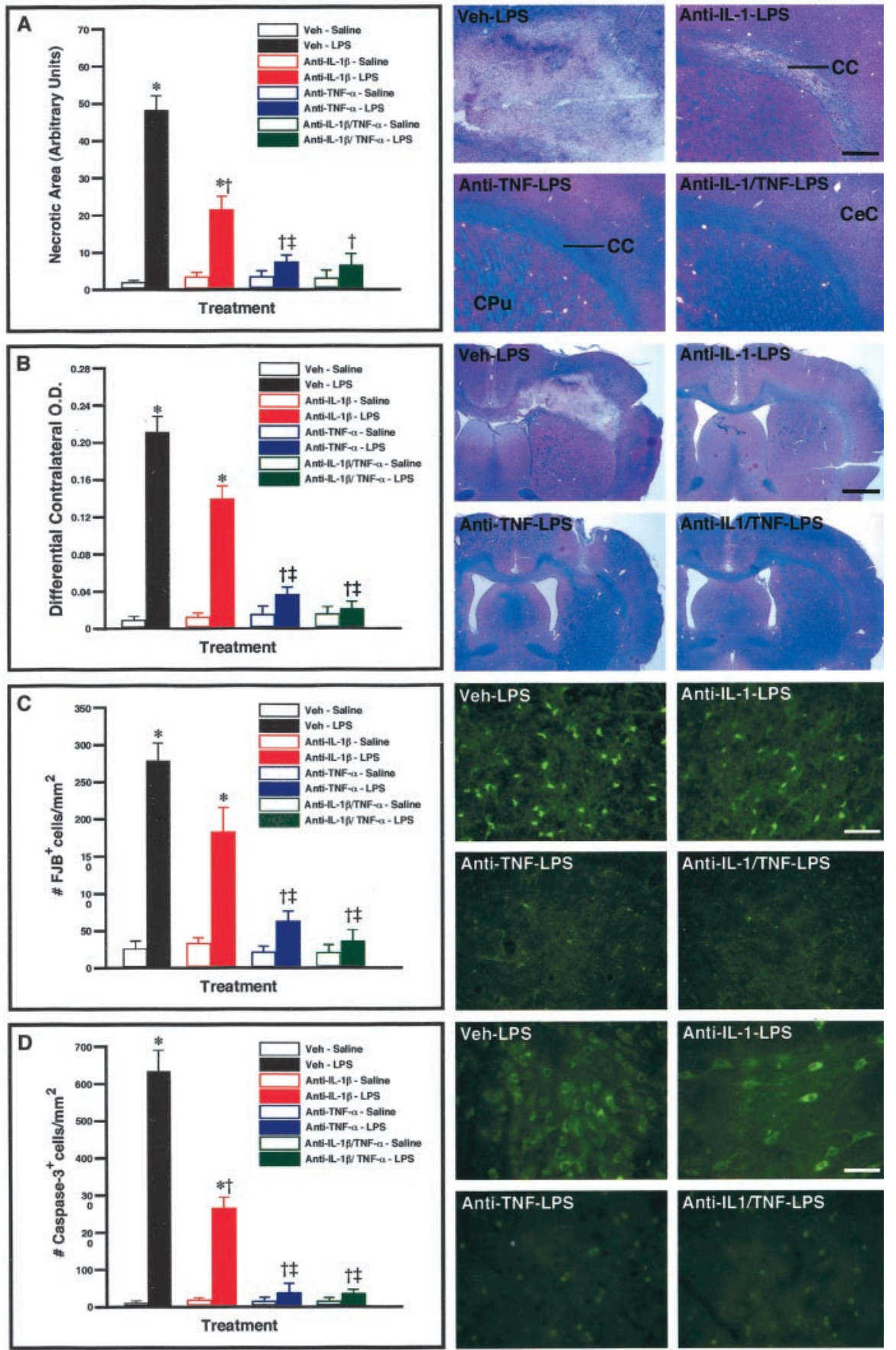

Figure 3. Role of interleukin $1 \beta$ (IL-1 $\beta$ ) and tumor necrosis factor $\alpha$ (TNF- $\alpha$ ) in the neurotoxic effects of lipopolysaccharide (LPS) in glucocorticoid receptor-deficient rats. Solutions containing an rlL-1 $\beta$ neutralizing antibody $(2 \mu \mathrm{g} / 5 \mu \mathrm{l}$ for $2 \mathrm{~min})$, an rTNF- $\alpha$ neutralizing antibody ( $2 \mu \mathrm{g} / 5 \mu \mathrm{l}$ for $2 \mathrm{~min}$.), a mixture of both neutralizing antibodies ( $4 \mu \mathrm{g} / 5 \mu \mathrm{l}$ for $2 \mathrm{~min}$ ), or vehicle were infused into the dorsal basal ganglia $10 \mathrm{hr}$ before the endotoxin challenge via the same route. These neutralizing antibodies were infused a second time just after the cerebral LPS treatment $(5 \mu \mathrm{g} / 2 \mu \mathrm{lfor} 2 \mathrm{~min})$, and rats were killed 7 d later. Please notethat all the animals included in this analysis were treated with RU486 (50 mg/kg per $200 \mu \mathrm{l}) 12 \mathrm{hr}$ before the LPS bolus to cause neurodegeneration. The necrotic area, extent of demyelination, number of Fluoro-Jade B (\#FJB) neurons, and number of cleaved caspase-3-immunoreactive cells are depicted in $A-D$, respectively. The photomicrographs depict representative examples of the different histological preparations that were used to quantify the data. All photomicrographs weretaken at the same rostrocaudal level. Results are expressed as mean \pm SEM for two (Veh-Saline) or five animals per group. ${ }^{*} p<0.05$ from their corresponding saline-treated groups. ${ }^{\dagger} p<0.05$ from the Veh-LPS group. ${ }^{\ddagger} p<0.05$ from the anti-lL- $1 \beta$-LPS group. CC, Corpus callosum; $C$ C C, cerebral cortex; $C P u$, caudoputamen. Magnification: $A, 10 \times ; B, 3.125 \times ; C, D, 100 \times$. Scale bars: $A, 125$ $\mu \mathrm{m} ; B, 1625 \mu \mathrm{m} ; C, D, 50 \mu \mathrm{m}$.

ganglia, hippocampal formation, cerebral cortex, and fibers of the corpus callosum. Finally, the marker of neuronal degeneration, FJB, remained undetectable in the brain of LPS-challenged animals.
This immune reaction, however, surprisingly became highly toxic for the cerebral tissue in rats pretreated with the GCR inhibitor RU486. The ability of GCs to modulate the innate immune response in the brain was expected, because these hormones are the most potent negative regulators of the proinflammatory signal transduction pathways and gene expression in macrophages and other systemic antigen-presenting cells (Webster et al., 2002). Although the intensity of the hybridization signal for most of the genes assessed was not necessarily stronger in the brain of LPS-injected rats pretreated with RU486, the signal remained positive for a longer period. This increase in the duration of the innate immune response therefore may lead to inappropriate levels of cytokines in the cerebral environment, the most critical one being TNF- $\alpha$. This cytokine is essentially produced by microglial cells in response to LPS, and GCs control the microglial expression of TNF- $\alpha$ (Nadeau and Rivest, 2002). Without this negative feedback, this cytokine becomes highly toxic and causes a nonselective cell death. These data, together with the inability of LPS to trigger apoptosis and necrosis in intact animals, indicate that the endotoxin is not by itself responsible for the neurodegeneration, but the microglial reactivity and uncontrolled production of TNF- $\alpha$ are the direct causes of the cerebral injury (Fig. 6).

Of interest is the nonspecific tissue destruction by the single LPS bolus in rats that received the GCR inhibitor. The same phenomenon took place in the brain after the chronic administration of TNF, but the cytokine took longer than LPS to damage the brain. Indeed, although the wound was already present $36 \mathrm{hr}$ after the combined treatment of LPS and RU486 and this effect was abolished by inhibiting the biological activity of the cytokine, the first signs of degeneration were found only between 3 and $7 \mathrm{~d}$ of chronic TNF infusion. It is possible that the concentration selected to deliver the cytokine chronically does not reflect the levels reached in the extracellular milieu when the endotoxin was injected in GC-deficient rats. The concentrations of TNF are actually determinant for the shift between cell survival and cell death (Sugarman et al., 1985), and such equilibrium was seriously compromised by the lack of appropriate negative feedback of GCs. The effects of TNF- $\alpha$ also depend on the type of cell. The viability of three human carcinoma cell lines, ME-180 (cervical), BT-20 (breast), and MCF-7 (breast), was reduced by 50\% at doses of $120-$ $500 \mathrm{U}$ TNF- $\alpha / \mathrm{ml}$, whereas other cell lines remained relatively insen- 
sitive to the cytokine (Sugarman et al., 1985; Beg and Baltimore, 1996). In the present case, however, the cytokine was capable of causing degeneration of all types of cells at the site of infusion.

Other cytokines may explain the delay between the treatment of LPS with RU486 and chronic TNF- $\alpha$ infusion. In this regard, inhibition of IL- $1 \beta$ partially prevented the degeneration induced by the combined treatment of RU486 and intracerebral LPS. The binding of IL- $1 \beta$ to its cognate type I receptor leads to the formation of the IL-1 receptor-associated kinase (IRAK)/TNF receptor-associated factor 6 (TRAF6) complex, which recruits the general adaptor protein MyD88 (myeloid differentiation factor 88 ) and activates NIK (NF- $\kappa \mathrm{B}$-inducible kinase)/IKK $(\mathrm{I} \kappa \mathrm{B} \alpha$ kinase) kinases involved in the phosphorylation and degradation of $\mathrm{I} \kappa \mathrm{B} \alpha$ (inhibitory factor $\kappa \mathrm{B} \alpha$ ) (Kawai et al., 1999). NF- $\kappa \mathrm{B}$ is then translocated into the nucleus and may bind to its $\kappa \mathrm{B}$ consensus sequence on target genes (Ghosh and Karin, 2002). Despite the beneficial influence of IL- $1 \beta$ during the innate immune response, there is accumulating evidence that this cytokine plays a key role in the neuroinflammatory response that may lead to cell death in neurodegenerative conditions (for review, see Allan and Rothwell, 2001). Compared with shortterm gliosis, constant activation of astrocytes and microglia by chronic autocrine and paracrine secretion of IL- $1 \beta$ inhibits re-outgrowth processes and promotes neurodegeneration (Streit et al., 1999). For instance, high levels of IL-1 $\beta$ or chronic induction of the proinflammatory cytokine can trigger neuronal cell death, which can be attenuated by IL-1 receptor antagonist (Rothwell and Luheshi, 2000).

The dichotomy of activated astrocytes and microglia as impediments or facilitators of CNS recovery is a common concept in the fields of regeneration and inflammation research. Studies havepointed out that the beneficial or noxious effects of astrogliosis and microgliosis are dependent on the duration of activation of glial cells (Streit et al., 1999). A short activation of microglial and astroglial cells after neuronal injury favors neuronal regeneration processes, and it confers protection to neurons through induction of neurotrophic factors, calcitonin generelated peptide, and ATP (Streit et al., 1999; Herx et al., 2000;
Veh
(14 days)

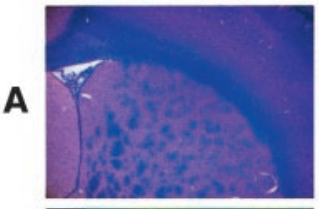

B

C

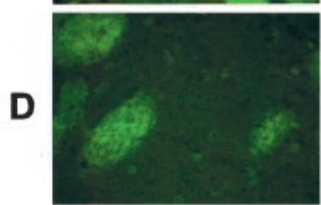

E

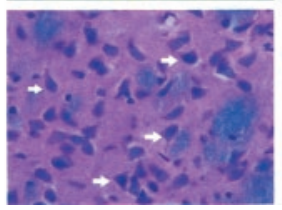

TNF- $\alpha$ (3 days)
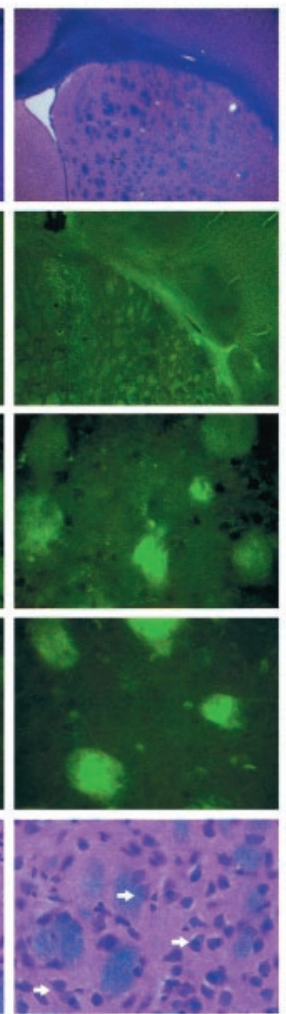

TNF- $\alpha$ (7 days)
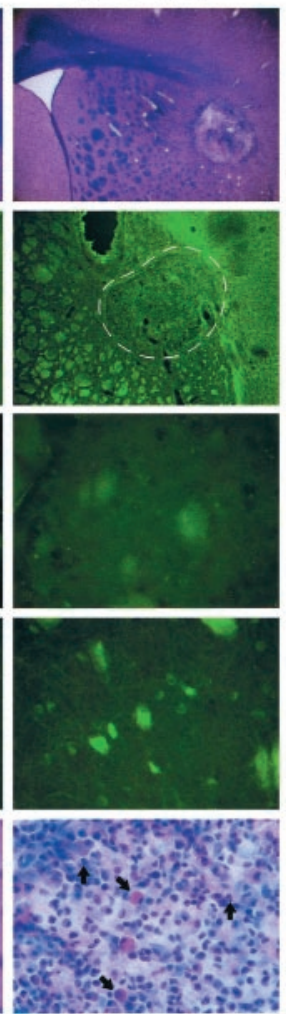

TNF- $\alpha$ (14 days)
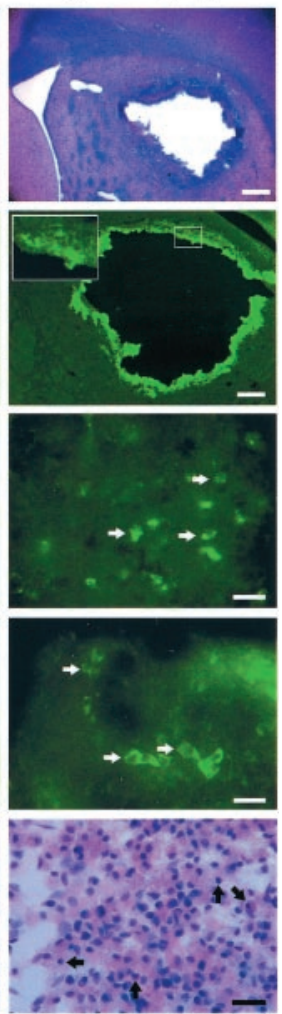

Figure 4. Chronic infusion of tumor necrosis factor $\alpha$ (TNF- $\alpha)$ induces cerebral damage. The animals received a chronic infusion with either the vehicle solution or rrTNF- $\alpha(0.0052 \mu \mathrm{g} / 0.26 \mu \mathrm{l}$ per hour $)$ and were killed $3,7,14$, and $21 \mathrm{~d}$ after implantation of the mini-osmotic pumps. $A$, Panels depict representative examples of demyelination and necrotic tissues stained with Luxol Fast Blue. B, Panels show examples of Fluoro-Jade B staining used here as an index of neurodegeneration. Apoptotic cells are depicted by the images in ( (DNA fragmentation) and $D$ (immunoreactive cleaved caspase- 3 cells). The striosome-matrix organization of the striatum explains the unspecific staining for both terminal deoxynucleotidyl transferase-mediated biotinylated UTP nick end over the striosomes is not cell specific and is attributable to anatomical properties of these structures. They can be detected with most staining procedures, including $\mathrm{LFB}(A, E)$. E, Panels show the progressive destruction of the extracellular matrix, modification same rostrocaudal level. Black arrows indicate infiltrating cells; white arrows indicate neurons. Magnification: $A, B, 3.125 \times ; C-E$ $100 \times$ Scale bars: $A, B, 1000 \mu \mathrm{m} ;(-E, 25 \mu \mathrm{m}$.

Nguyen et al., 2002). It is of interest to note that microglial IL-1 $\beta$ is the key molecule involved in the release of specific growth factors in response to cerebral injuries (Herx et al., 2000), and inhibition of this cytokine failed to alter the progress of motor neuron degeneration in the mouse model of amyotrophic lateral sclerosis (Nguyen et al., 2001). The dual nature of IL-1 in the CNS may therefore depend on the type of injuries and insults. In spite of this, the cerebral production of IL- 1 is not an absolute prerequisite in the cytotoxic effects of LPS, whereas the cerebral tissue remained essentially intact in animals that received anti-TNF- $\alpha$ before the LPS challenge.

Table 3. Quantification of brain damage induced by chronic injection of either vehicle solution (Veh) or TNF- $\alpha$

\begin{tabular}{lccccc}
\hline & Condition & & & \\
\cline { 2 - 5 } & Veh (14 d) & TNF- $\alpha$ (3d) & TNF- $\alpha$ (7 d) & TNF- $\alpha$ (14 d) \\
\hline Demyelination (differential 0.D.) & $0.007 \pm 0.001$ & $0.009 \pm 0.002$ & $0.033^{*} \pm 0.012$ & $0.059^{*} \pm 0.026$ & TNF- $\alpha$ (21 d) \\
Necrotic area (arbitrary units) & $0 \pm 0$ & $0 \pm 0$ & $14.36^{*} \pm 2.71$ & $26.28^{*} \pm 4.78$ & $23.64^{*} \pm 0.031$ \\
TdT-FragEL (number of cells $/ \mathrm{mm}^{2}$ ) & $0 \pm 0$ & $0 \pm 0$ & $0 \pm 0$ & $137^{*} \pm 23$ & $129^{*} \pm 34$ \\
Cleaved caspase-3 (number of cells $/ \mathrm{mm}^{2}$ ) & $0 \pm 0$ & $0 \pm 0$ & $0 \pm 0$ & $154^{*} \pm 29$ & $137^{*} \pm 36$ \\
\hline
\end{tabular}

Veh, Chronic injection of vehicle solution; TNF- $\alpha$, chronic injection of tumor necrosis factor $\alpha$; ${ }^{*} p 0.05$ from their corresponding vehicle-treated groups. 


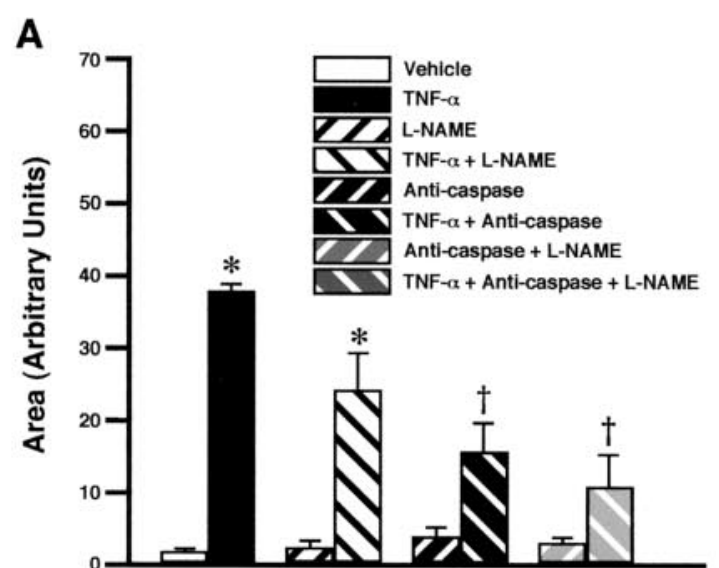

B

Treatment

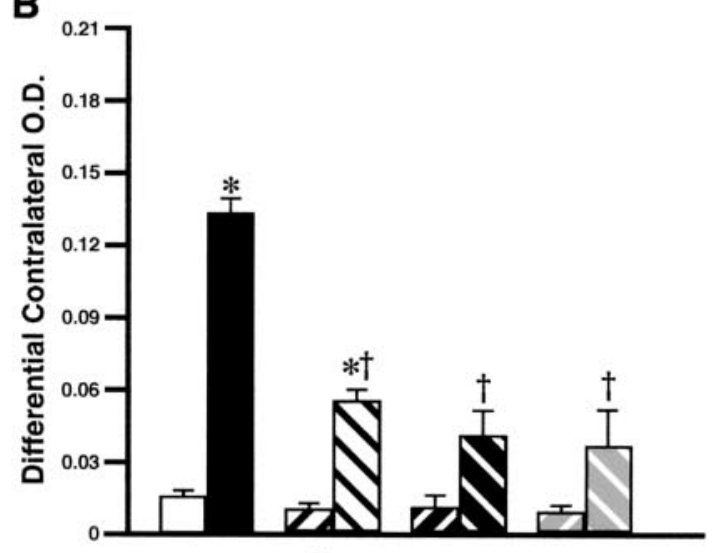

C

Treatment

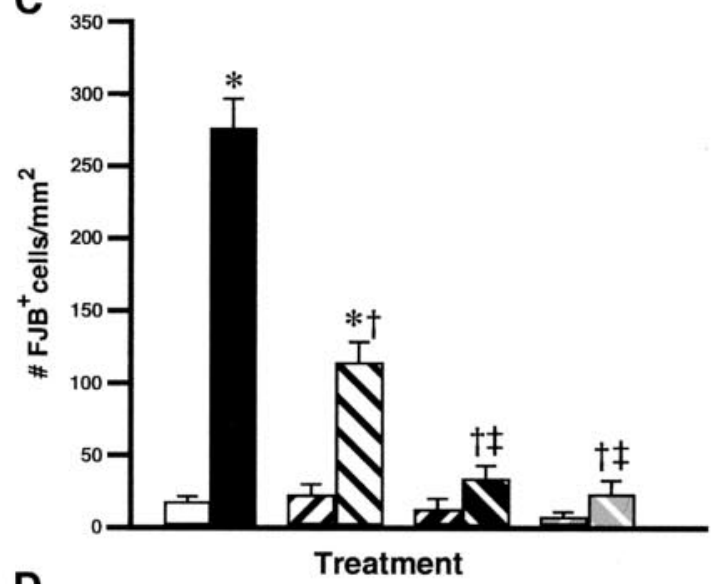

D

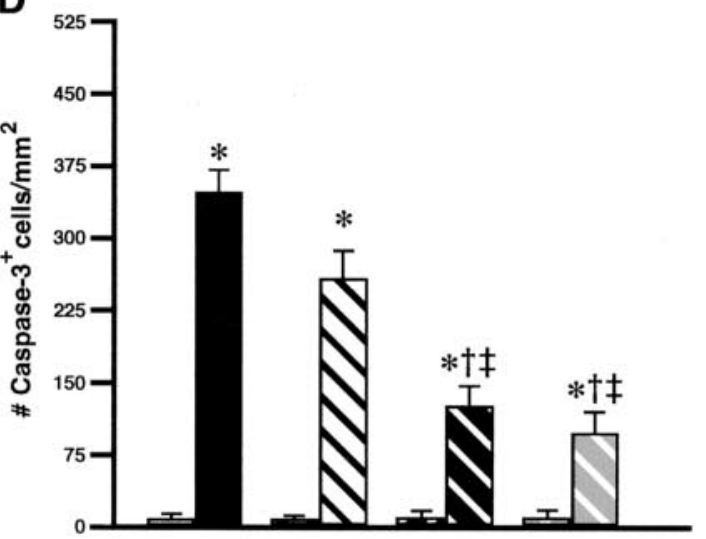

Treatment
The binding of TNF- $\alpha$ to its cognate receptors leads to the formation of the TNF-R1-associated death domain (TRADD)/ TRAF2 complex, which activates the NF- $\kappa \mathrm{B}$ signaling events. TNF- $\alpha$ is actually one of the most potent effectors of NF- $\kappa$ B activity through the $55 \mathrm{kDa}$ TNF type I receptor in most types of cells in the systemic immune system as well as in the CNS (Laflamme and Rivest, 1999). Fas-associated death domain protein (FADD)/mediator of receptor-induced toxicity 1 (MORT1), TRAF2, and the death domain kinase receptor interacting protein (RIP) are recruited and may also interact directly with TRADD (Siegel and Lenardo, 2001). While FADD/MORT1 is essential for TNF-induced apoptosis, RIP and TRAF2 seem to be the key molecules for activating both NF- $\kappa \mathrm{B}$ and MAP kinases (Chan et al., 2000). The latter is a major survival pathway (Karin and Lin, 2002); it leads to the synthesis of several anti-apoptotic proteins and inhibition of NF- $\kappa \mathrm{B}$ provokes cell death in the presence of TNF- $\alpha$ (Beg and Baltimore, 1996; Van Antwerp et al., 1996; Wang et al., 1996). However, the increase in the activity of this pathway by RU486 clearly failed to prevent the neurotoxic effects of the cytokine. These data, together with the robust immunoreactive signal for the cleaved caspase- 3 and caspase inhibition that significantly prevented the cellular degeneration caused by the chronic TNF- $\alpha$ infusion, support the concept that apoptotic signaling events circumvented the protecting activity of NF- $\kappa$ B.

Other mechanisms are nevertheless involved, because caspase inhibition did not completely abolish the neurotoxic effects of TNF- $\alpha$. Although the drugs used in this study may not be totally effective in preventing the activities of the caspases, degeneration by both necrosis and apoptosis was found in response to an inappropriate production of TNF- $\alpha$. Nitric oxide plays a role in this regard but only partially, because the nonselective NOS inhibitor L-NAME had a limited protective role in TNF-induced cell death. Future studies are therefore essential to clarify the exact intracellular mechanisms by which the innate immune system can have the ability to dramatically kill cells of the brain in the absence of an adequate binding of GCs to their nuclear receptors. It is tempting to propose that inappropriate negative control of GCs on the inflammatory events in the CNS may be a common mechanism for the initiation of neurodegenerative disorders that have an immune etiology.

In summary, the inflammatory response lasted longer in the brain of animals that received the GR antagonist RU486 before the intracerebral LPS infusion. Surprisingly, a single bolus of LPS caused a rapid and severe neurodegeneration in RU486pretreated animals. This neurotoxic effect of LPS was essentially abolished by inhibiting the biological activity of TNF- $\alpha$ in the CNS. Finally, we show that TNF- $\alpha$-induced neurodegeneration involves both caspase and NO pathways. These data provide first-

Figure 5. Partial role of nitric oxide (NO) and caspase pathways in the neurotoxic effects of TNF- $\alpha$ chronically infused into the rat brain. The mini-osmotic pumps were filled with vehicle solution, the nonselective inhibitor of NOS L-NAME $(4.167 \mu \mathrm{g} / 0.26 \mu \mathrm{l}$ per hour), a mixture containing caspase- 2 and caspase- 8 inhibitors $(3.125 \mu \mathrm{g} / 0.26 \mu \mathrm{l}$ per hour), or a mixture of the three drugs with and without TNF- $\alpha(0.0052 \mu \mathrm{g} / 0.26 \mu \mathrm{l}$ per hour). The animals were killed $14 \mathrm{~d}$ after the beginning of the infusion in the dorsal basal ganglia. The necrotic area, extent of demyelination, number of Fluoro-Jade B (\# FJB) neurons, and number of cleaved caspase-3immunoreactive cells are depicted in $A-D$, respectively. Results are expressed as mean \pm SEM for two (Vehicle) or four animals per group. Data were analyzed by a two-way ANOVA, followed by a Bonferroni/Dunn test procedure as post hoc comparison (Statview 4.01). ${ }^{*} p<0.05$ from their corresponding vehicle-treated groups. ${ }^{\dagger} p<0.05$ from the $\operatorname{rrTNF}-\alpha$-treated group. ${ }^{\ddagger} p<$ 0.05 from the L-NAME + rrTNF- $\alpha$-treated group. 
time evidence that without endogenous GCs, the innate immune response and the resulting overproduction of TNF- $\alpha$ have serious detrimental consequences for the brain.

\section{References}

Allan SM, Rothwell NJ (2001) Cytokines and acute neurodegeneration. Nat Rev Neurosci 2:734-744.

Beg AA, Baltimore D (1996) An essential role for NF-kappaB in preventing TNF-alphainduced cell death. Science 274:782-784.

Chan FK, Siegel RM, Lenardo MJ (2000) Signaling by the TNF receptor superfamily and T cell homeostasis. Immunity 13:419-422.

De Bosscher K, Schmitz ML, Vanden Berghe W, Plaisance S, Fiers W, Haegeman G (1997) Glucocorticoid-mediated repression of nuclear factor-kappaB-dependent transcription involves direct interference with transactivation. Proc Natl Acad Sci USA 94:13504-13509.

Ghosh S, Karin M (2002) Missing pieces in the NF-kappaB puzzle. Cell [Suppl] 109:S81-96.

Herx LM, Rivest S, Yong VW (2000) Central nervous system-initiated inflammation and neurotrophism in trauma: IL-1beta is required for the production of ciliary neurotrophic factor. J Immunol 165:2232-2239.

Karin M, Lin A (2002) NF-kappaB at the crossroads of life and death. Nat Immunol 3:221-227.

Kawai T, Adachi O, Ogawa T, Takeda K, Akira S (1999) Unresponsiveness of MyD88deficient mice to endotoxin. Immunity $11: 115-122$

Laflamme N, Rivest S (1999) Effects of systemic immunogenic insults and circulating proinflammatory cytokines on the transcription of the inhibitory factor kappa B alpha within specific cellular populations of the rat brain. J Neurochem 73:309-321.

Laflamme N, Rivest S (2001) Toll-like receptor 4: the missing link of the cerebral innate immune response triggered by circulating gram-negative bacterial cell wall components. FASEB J 15:155-163.

Laflamme N, Lacroix S, Rivest S (1999) An essential role of interleukin- $1 \beta$ in mediating NF- $\kappa \mathrm{B}$ activity and COX-2 transcription in cells of the blood-brain barrier in response to systemic and localized inflammation, but not during endotoxemia. J Neurosci 19:10923-10930.

Laflamme N, Souci G, Rivest S (2001) Circulating cell wall components derived from gram-negative and not gram-positive bacteria cause a profound transcriptional activation of the gene encoding toll-like receptor 2 in the CNS. J Neurochem 70:648-657.

McKay LI, Cidlowski JA (1999) Molecular control of immune/inflammatory responses: interactions between nuclear factor-kappa B and steroid receptor-signaling pathways. Endocr Rev 20:435-459.

Nadeau S, Rivest S (2000) Role of microglial-derived tumor necrosis factor in mediating $\mathrm{CD} 14$ transcription and NF- $\kappa \mathrm{B}$ activity in the brain during endotoxemia. J Neurosci 20:3456-3468.

Nadeau S, Rivest S (2001) The complement system is an integrated part of the natural innate immune response in the brain. FASEB J 15:1410-1412.

Nadeau S, Rivest S (2002) Endotoxemia prevents the cerebral inflammatory wave induced by intraparenchymal lipopolysaccharide injection: role of glucocorticoids and CD14. J Immunol 169:3370-3381.

Nguyen MD, Julien JP, Rivest S (2001) Induction of proinflammatory molecules in mice with amyotrophic lateral sclerosis: no requirement for proapoptotic interleukin-1beta in neurodegeneration. Ann Neurol $50: 630-639$
Nguyen MD, Julien JP, Rivest S (2002) Innate immunity: the missing link in neuroprotection and neurodegeneration? Nat Rev Neurosci 3:216-227.

Rothwell NJ, Luheshi GN (2000) Interleukin 1 in the brain: biology, pathology and therapeutic target. Trends Neurosci 23:618-625.

Sapolsky RM (1996) Why stress is bad for your brain. Science 273:749-750.

Siegel RM, Lenardo MJ (2001) To B or not to B: TNF family signaling in lymphocytes. Nat Immunol 2:577-578.

Streit WJ, Walter SA, Pennell NA (1999) Reactive microgliosis. Prog Neurobiol 57:563-581.

Sugarman BJ, Aggarwal BB, Hass PE, Figari IS, Palladino MA Jr, Shepard HM (1985) Recombinant human tumor necrosis factor-alpha: effects on proliferation of normal and transformed cells in vitro. Science 230:943-945.

Thibeault I, Laflamme N, Rivest S (2001) Regulation of the gene encoding the monocyte chemoattractant protein $1(\mathrm{MCP}-1)$ in the mouse and rat brain in response to circulating LPS and proinflammatory cytokines. J Comp Neurol 434:461-477.

Van Antwerp DJ, Martin SJ, Kafri T, Green DR, Verma IM (1996) Suppression of TNF-alpha-induced apoptosis by NF-kappaB. Science 274:787-789.

Wang CY, Mayo MW, Baldwin AS Jr (1996) TNF- and cancer therapyinduced apoptosis: potentiation by inhibition of NF-kappaB. Science 274:784-787.

Webster JI, Tonelli L, Sternberg EM (2002) Neuroendocrine regulation of immunity. Annu Rev Immunol 20:125-163.

Wissink S, van Heerde EC, vand der Burg B, van der Saag PT (1998) A dual mechanism mediates repression of NF-kappaB activity by glucocorticoids. Mol Endocrinol 12:355-363. 